\title{
Innovación en los gobiernos locales en Iberoamérica. Posibilidades a partir de la nueva gerencia pública
}

\author{
JAIME TORRES FRAGOSO, Ph.D., ${ }^{1,2}$ \\ Jefe de Licenciatura en Administración pública y profesor investigador, Universidad del Istmo, México. \\ jaimetorres@bianni.unistmo.edu.mx
}

\begin{abstract}
RESUMEN
El artículo pretende demostrar, a través de la revisión de tres estudios de caso, que los gobiernos locales de Latinoamérica vienen desarrollando interesantes programas de innovación y que éstos se sustentan en el modelo de Nueva Gerencia Pública (NGP). Para lograr ese objetivo, se presentan las características de la innovación, los rasgos que definen la gestión de los gobiernos locales y los elementos de la NGP. Los resultados señalan que las posibilidades para construir mejores programas y servicios públicos son amplias, y que la NGP representa un modelo referencial valioso para enfrentar ese reto. Finalmente, se recomienda concebir este tipo de herramientas administrativas como medios en el gran proyecto de ubicar al ciudadano en el centro de la acción gubernamental.
\end{abstract}

Palabras clave. Gobiernos locales; innovación; nueva gerencia pública.

Recibido: 22-jun-11, corregido: 27-abr-12 y aceptado: 26-jun-12

Clasificación JEL: D73; H11; H70

@

1 Este documento fue seleccionado en la convocatoria para enviar artículos, Call for Papers, realizada en el marco del “II Simposio Iberoamericano de Estudios Gerenciales: Una mirada interdisciplinar a la innovación”, organizado por la revista académica Estudios Gerenciales bajo la dirección de la Facultad de Ciencias Administrativas y Económicas de la Universidad Icesi; el evento tuvo lugar los días 12, 13 y 14 de octubre de 2011, en la ciudad de Cali (Colombia). Este documento fue presentado en las sesiones simultáneas del área de "Innovación en políticas públicas".

2 Dirigir correspondencia a: Carretera a Chihuitán s/n, Ixtepec, Oaxaca, México, C.P. 70110. 


\section{Local government innovation in Ibero-American countries. New public management inspires possibilities}

This article reviews three different case studies with the aim to demonstrate that local governments in Latin America have been developing interesting innovation programs based on a new public management (NPM) model. To this end, it provides a description of the characteristics of innovation, the features of local government management, and the fundamentals of the NPM model. The results reveal that there are extensive possibilities for designing better programs and public services and, at the same time, confirm that the NPM model is a valuable framework that can be used for dealing with this challenge. Lastly, it provides a recommendation to view these kinds of management tools as instruments to make progress with the important project of placing citizens at the center of governmental actions.

Keywords. Local governments; innovation; new public management.

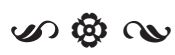

\section{Inovação nos governos locais na América Latina. Possibilidades a partir da nova gestão pública}

O artigo procura demonstrar, através da análise de três estudos de caso, que os governos locais na América Latina têm desenvolvendo programas inovadores interessantes que se baseiam no modelo da Nova Gestão Pública (NGP). Para atingir este objetivo, apresentamos as características da inovação, as características que definem a gestão do governo local e os elementos do NGP. Os resultados mostram que as possibilidades para construir programas melhores e serviços públicos são amplas, e que o NGP representa um modelo de referência valiosa para enfrentar este desafio. Finalmente, recomendamos conceder este tipo de ferramentas administrativas, tais como meios no grande esquema de colocar o cidadão no centro da ação do governo.

Palavras-chave. Os governos locais; a inovação; a nova gestão pública. 


\section{Introducción}

Desde la puesta en marcha de los estudios de opinión, la ciudadanía de los países de la región latinoamericana se han mostrado insatisfecha con el desempeño de sus gobiernos, particularmente, en lo que respecta a la provisión de servicios públicos como el transporte, educación, salud, vivienda, seguridad, alumbrado, drenaje, agua y alcantarillado, limpieza de calles y recolección de basura, etc. No obstante, en los últimos años se ha experimentado avances, tanto en la preocupación de los gobiernos por generar documentos que representan compromisos para brindar mejores servicios y construir una mejor gestión pública, como en la implementación de mejores prácticas gerenciales en su interior.

Es posible suponer que buena parte de esas iniciativas y experiencias se han inspirado en la filosofía administrativa denominada como Nueva Gerencia Pública (NGP), la que se convirtió en un referente para la reforma del sector público con carácter global, patentada en países angloparlantes pero difundida en todos los aparatos gubernamentales de occidente, incluyendo a Iberoamérica. Entre las ideas principales de la NGP, las cuales descansan en el concepto de innovación organizacional, destacan la ejecución de programas más efectivos y mejoramiento de metas, operaciones más eficientes, la mejora de la calidad, la creación de estructuras organizacionales más flexibles, la incorporación más vigorosa del capital privado en la prestación de servicios públicos y la creación de medios más competitivos y de mercado dentro de las organizaciones públicas (Shand, 1996).

Con base en lo anterior, el propósito central de la presente investigación es el de mostrar que en fechas recientes, al interior de los gobiernos regionales y municipales de América Latina, se han implementado planes y programas de diversa naturaleza (tecnológica, operativa, medioambiental, financiera, etc.) que, por su concepción e impactos, se pueden considerar como innovadores y cuya finalidad es la de mejorar en forma significativa la gestión pública y por ende la calidad de vida de la ciudadanía. Con el logro de esta meta, el artículo pretende asimismo identificar algunas de las posibilidades para la innovación en los gobiernos locales iberoamericanos, así como aquellos elementos fundamentales para su implementación. Otros objetivos del trabajo, cuyo cumplimiento permitirá alcanzar y explicar de mejor manera el primero, son los de presentar los rasgos de los gobiernos desde una perspectiva organizacional, haciendo énfasis en el nivel local; revisar el concepto, los tipos y niveles de la innovación en las organizaciones; exponer las ideas centrales del modelo de NGP; además de identificar y analizar tres experiencias innovadoras de gestión pública en gobiernos locales.

La hipótesis de trabajo es que la innovación es una actividad altamente factible y en ascenso al interior de los gobiernos locales iberoamericanos y que, además, este proceso se impulsa y apoya en el modelo de nueva gerencia pública. En este sentido, se estima que el manejo y la difusión de ideas acerca de la NGP como variable independiente, estaría generando más programas gubernamentales innovadores.

A partir de lo que diversos autores identifican como enfoque cualitativo, ${ }^{3}$ esta investigación incorpora un diseño no expe-

3 Para Hernández, Fernández \& Baptista (2007, p. 9), "el enfoque cualitativo puede definirse como un conjunto de prácticas interpretativas que hacen al mundo visible, lo transforman y convierten en una serie de representaciones en forma de observaciones, anotaciones, grabaciones y documentos". Se subraya que es interpretativo al dar sentido a los fenómenos con base a los significados que los estudiosos les den. 
rimental (se desarrolla sin manipular intencionalmente variables) tipo transeccional (por medio de la recolección de datos en un momento único) puramente descriptivo. La estrategia metodológica empleada se integra a partir de dos ejes de acción, desarrollados en forma paralela, y que engloban sus correspondientes actividades. El primero de ellos representa el sustento teórico del trabajo y consistió en la investigación documental y análisis de las ideas y desarrollos en torno a cuatro elementos: 1. Los rasgos de los gobiernos regionales y municipales en Iberoamérica y México, 2. Las características de la innovación administrativa, 3. Los conceptos que sustentan el modelo de NGP, y 4. La incorporación de la filosofía de la NGP en América Latina tomando como referencia algunos de los documentos que el Centro Latinoamericano de Administración para el Desarrollo (CLAD) ha elaborado con tal propósito en años recientes.

El segundo eje tiene un talante más orientado a la gestión pública aplicada y se integró por las siguientes etapas: 1. Identificación, a partir de un universo muy amplio de experiencias disponibles, de estudios de caso relativos a programas impulsados por los gobiernos locales de la región, que se pudieran considerar como exitosos con base en su originalidad, los objetivos planteados y los resultados alcanzados; 2. Del universo de casos exitosos identificados, se seleccionaron tres que por su alcance y nivel de impacto, se estimó permitirían explicar de manera adecuada las posibilidades de la innovación organizacional. ${ }^{4}$ Los casos selec-

4 Los estudios de caso sirven para identificar experiencias innovadoras y buenas prácticas de gestión (actividad conocida como investigación de casos), además de dar a conocer esas experiencias a estudiantes, académicos y servidores públicos. En tal sentido, se considera que el método del caso es útil en transmitir conocimientos a través de técnicas de aprendizaje activo, en disciplinas orientadas a la acción, tal como la gestión pública (Nava, cionados fueron los siguientes: el Servicio de Atención al Ciudadano (sac) de Bahía, Brasil; el proyecto de la Ciudad Rural Sustentable (CRS) Nuevo Juan del Grijalva, en Chiapas, México; además del programa EcoImpulso (EI), de Tepatitlán, Jalisco, también de México; 3. Los programas seleccionados fueron estudiados minuciosamente de tal forma que se pudieran identificar sus metas planteadas, las estrategias adoptadas, los subprogramas que los conforman, así como los resultados alcanzados; y 4 . Finalmente y con la pretensión de poder entender y explicar esos resultados, varios de los logros e impactos registrados en los estudios de caso se contrastaron con la teoría tanto de la innovación como de la NGP.

En correspondencia con los objetivos y la metodología descrita, el cuerpo del documento se compone de cuatro partes. En la primera se exponen las características de los gobiernos locales desde una perspectiva organizacional. En forma complementaria, se presentan algunas de las diferencias más significativas entre la gestión pública y privada, además de un breve análisis de los principales problemas de los gobiernos municipales, haciendo énfasis en el caso mexicano. En la segunda se estudia el concepto de innovación organizacional, junto a sus tipos y categorías, y las características de las organizaciones innovadoras. La tercera parte analiza las características y componentes de la NGP y de las organizaciones posburocráticas en comparación con los rasgos que han definido tradicionalmente la administración pública iberoamericana, asociados al modelo burocrático, las razones por las que la NGP se considera un modelo innovador para la administración pública, y los documentos inspirados en ella para tratar de acceder a una gestión pública más

2000). Con toda la proporción guardada, este trabajo pretende también alcanzar estos fines. 
digna y profesional en Iberoamérica. En la cuarta sección se presenta el análisis de las experiencias seleccionadas. Por último, en la sección de conclusiones, el trabajo incorpora una reflexión acerca de los resultados obtenidos, así como de las alternativas y posibilidades para la innovación en los gobiernos regionales a partir de la NGP.

\section{Los gobiernos locales: organizaciones complejas}

Tal y como bien lo apunta Tamayo (1997), los gobiernos son en esencia grupos de organizaciones de diversa índole: oficinas de tramitación y cobro, escuelas, clínicas de salud, departamentos de policía, juzgados, empresas paraestatales, áreas de registro civil, entre muchas otras. Como cualquier otra organización, están inmersas en un proceso productivo, ya que transforman recursos (inputs) humanos, financieros y tecnológicos en bienes o servicios (outputs), a través de los cuales deberán satisfacer necesidades y problemas públicos y con ello lograr impactos globales de carácter social, político y económico (outcomes), lo que el Banco Interamericano para el Desarrollo -BID y el CLAD (2007) identifican como valor público. Como complemento a lo anterior, Arellano \& Cabrero (2000) indican que los gobiernos reúnen las siguientes tres características: 1. El gobierno como estructuras organizacionales. Coincidiendo con Tamayo, se plantea que los gobiernos no son monolitos abstractos, por el contrario, se conforman de una amplia variedad de organizaciones diferentes en su tamaño, misión, estrategias, recursos, estructura y contexto.

2. La dualidad de las organizaciones gubernamentales. Este rasgo se explica a partir que los organismos estatales ${ }^{5}$ son formal

5 Dado que las agencias gubernamentales forman parte del Estado y que la administración pública es un componente del gobierno, en este documento se emplearán y legalmente predeterminados para su surgimiento (nacen por decreto oficial) y en lo que atañe a sus objetivos. Al mismo tiempo, una vez que se han constituido, adquieren vida propia, ya que se enfrentan a un medio ambiente o contexto específico con sus propios recursos, lo que generará un esfuerzo tendiente a crear capacidades para su supervivencia.

3. El gobierno como red de organizaciones. $\mathrm{Al}$ nacer, las agencias públicas se insertan en una red ya existente de otras organizaciones gubernamentales, con las cuales mantienen una amplia relación en materia fiscal, subvenciones, regulación, operativa, etc., lo anterior con independencia de la propia dinámica de relaciones al interior de cada organización. ${ }^{6}$

Además de lo anterior se debe considerar que, en comparación con la gestión de las organizaciones privadas (empresas), las pertenecientes a la administración pública tienen un horizonte de tiempo más corto, sus mecanismos de medición de desempeño no son tan claros, el manejo del personal es más rígido, están sujetas a un mayor escrutinio normativo y legal, y están más expuestas a los medios de comunicación. Estas y otras diferencias se aprecian en la Tabla 1.

A ello hay que agregar que los gobiernos locales iberoamericanos, sean éstos de carácter regional o local (principalmente), históricamente se han manejado con menos capacidad financiera y operativa que los gobiernos centrales o federales para responder a las exigencias que su propio quehacer les demanda. Al respecto, Cabrero (2007), refiriéndose al caso mexicano, ha señalado que

indistintamente los términos estatal, gubernamental y de la administración pública para referirse a sus diversas organizaciones.

6 Este fenómeno puede ser comprendido a partir del análisis ejecutado por autores como Agranoff (1997) sobre las Relaciones Intergubernamentales (RIG) y la Gestión Intergubernamental (GIG). 
Tabla 1. Diferencias de la gestión pública y privada

\begin{tabular}{|c|c|c|c|}
\hline No. & Indicador & Gestión pública & Gestión privada \\
\hline 1 & La perspectiva del tiempo & $\begin{array}{l}\text { Horizontes de tiempo breves, } \\
\text { sujetos a calendarios políticos }\end{array}$ & Perspectiva de más tiempo \\
\hline 2 & Duración & Relativamente breve & Periodo más largo \\
\hline 3 & Medición de desempeño & $\begin{array}{l}\text { Pocos acuerdos sobre los pará- } \\
\text { metros para evaluar }\end{array}$ & $\begin{array}{l}\text { Bien establecidas varias } \\
\text { pruebas de desempeño }\end{array}$ \\
\hline 4 & Limitaciones de personal & $\begin{array}{l}\text { La normatividad complica la } \\
\text { gestión de personal }\end{array}$ & $\begin{array}{l}\text { Tiene mucho mayor margen } \\
\text { de acción }\end{array}$ \\
\hline 5 & Igualdad y eficiencia & $\begin{array}{l}\text { Busca crear condiciones de } \\
\text { igualdad entre grupos }\end{array}$ & $\begin{array}{l}\text { Busca la eficiencia y el des- } \\
\text { empeño competitivo }\end{array}$ \\
\hline 6 & $\begin{array}{l}\text { Procesos públicos contra } \\
\text { procesos privados }\end{array}$ & Expuesta a la vista del público & $\begin{array}{l}\text { Más discreta, procesos me- } \\
\text { nos expuestos al público }\end{array}$ \\
\hline 7 & $\begin{array}{l}\text { Función de la prensa y } \\
\text { medios informativos }\end{array}$ & $\begin{array}{l}\text { Debe enfrentarse a la prensa y } \\
\text { otros medios informativos }\end{array}$ & $\begin{array}{l}\text { Sus decisiones aparecen } \\
\text { menos en la prensa }\end{array}$ \\
\hline 8 & Persuasión y dirección & $\begin{array}{l}\text { Los gestores públicos creen } \\
\text { que deben responder a muchos } \\
\text { superiores }\end{array}$ & $\begin{array}{l}\text { Los administradores priva- } \\
\text { dos tienden a buscar una } \\
\text { sola autoridad superior }\end{array}$ \\
\hline 9 & $\begin{array}{l}\text { Impacto legislativo y } \\
\text { judicial }\end{array}$ & $\begin{array}{l}\text { Se ve sometida a un minucioso } \\
\text { escrutinio de los poderes Legis- } \\
\text { lativo y Judicial }\end{array}$ & $\begin{array}{l}\text { Por lo común escapa a ese } \\
\text { tipo de escrutinio }\end{array}$ \\
\hline 10 & El objetivo final & $\begin{array}{l}\text { Rara vez tiene un objetivo final } \\
\text { claro }\end{array}$ & $\begin{array}{l}\text { Busca la ganancia, el desem- } \\
\text { peño y la supervivencia }\end{array}$ \\
\hline
\end{tabular}

Fuente: Adaptado de Allison (1999, pp. 754-757).

la mayoría de las administraciones municipales están aún en proceso de construcción de los sistemas administrativos básicos, ya que son frágiles en sus capacidades de acción, presentan debilidad institucional y niveles muy bajos de recursos y facultades. Sin embargo, como refiere el mismo autor, varios gobiernos municipales están implementando programas para mejorar su organización y gestión de servicios y otros más experimentan una etapa de modernización por medio de la realización de agudas transformaciones en sus procesos estratégicos, operativos y de servicio, que les hagan dejar la gestión burocrática tradicional y les acerque a una administración orientada al cliente, inspirada en las ideas de la NGP. La problemática del gobierno local mexicano se condensa en la Tabla 2.

\section{La innovación organizacional: concep- to, tipos y niveles}

Por innovación administrativa se puede entender el "proceso de crear nuevos o mejores bienes o servicios que los clientes desean o por el proceso de buscar nuevas formas de producirlos o proporcionarlos" (Jones \& George, 2006, p. 28). A partir de esta definición, es indispensable que los gobiernos locales emprendan amplios procesos de innovación que los lleven a flexibilizar sus estructuras, a mejorar la calidad de sus servicios y a tener una mejor relación con los agentes externos con los que guardan íntima relación. Para ese propósito, se pueden distinguir dos tipos de innovaciones organizacionales: la técnica y la administrativa (Cabrero, Arellano \& Amaya, 200o). 
Tabla 2. Los principales problemas del gobierno municipal en México

\begin{tabular}{|c|c|c|}
\hline No. & Problemática & Algunas evidencias \\
\hline \multirow[t]{2}{*}{1} & \multirow{2}{*}{$\begin{array}{l}\text { La gran mayoría de } \\
\text { los municipios mexi- } \\
\text { canos se caracteriza } \\
\text { por un muy débil } \\
\text { marco normativo y } \\
\text { reglamentario }\end{array}$} & $\begin{array}{l}64 \% \text { de los municipios carecen de un reglamento interno de la } \\
\text { administración municipal y en } 22 \% \text { no está actualizado. }\end{array}$ \\
\hline & & $\begin{array}{l}\text { En } 52 \% \text { de los municipios no hay un reglamento de obra pública y } \\
\text { en } 20 \% \text { no hay un plan de desarrollo. }\end{array}$ \\
\hline \multirow[t]{3}{*}{2} & \multirow{3}{*}{$\begin{array}{l}\text { Sistemas adminis- } \\
\text { trativos obsoletos } \\
\text { dominan el escenario } \\
\text { municipal }\end{array}$} & $\begin{array}{l}\text { En } 60 \% \text { de los municipios no existe un área especializada en la } \\
\text { evaluación y supervisión del gasto, tal como una oficialía mayor o } \\
\text { una dirección administrativa. }\end{array}$ \\
\hline & & En 65\% no hay un área de administración de personal. \\
\hline & & $\begin{array}{l}75 \% \text { reconocen haber recaudado impuestos y derechos ( } 75 \% \text { por } \\
\text { debajo de lo presupuestado). }\end{array}$ \\
\hline \multirow[t]{3}{*}{3} & \multirow{3}{*}{$\begin{array}{l}\text { Existe un débil nivel } \\
\text { de profesionalización } \\
\text { de gobernantes y } \\
\text { funcionarios muni- } \\
\text { cipales }\end{array}$} & $\begin{array}{l}18 \% \text { de los presidentes municipales no tienen estudios medios o } \\
\text { superiores en algún campo profesional. }\end{array}$ \\
\hline & & $\begin{array}{l}\text { La mitad de los alcaldes no tenía experiencia previa en la adminis- } \\
\text { tración pública. }\end{array}$ \\
\hline & & $\begin{array}{l}\text { Los funcionarios de la administración municipal tienen poca ex- } \\
\text { periencia en su función: } 30 \% \text { tienen menos de un año en el puesto, } \\
55 \% \text { menos de tres años y sólo } 5 \% \text { más de cinco años de antigüedad } \\
\text { en el puesto. }\end{array}$ \\
\hline
\end{tabular}

Fuente: Elaboración propia con base en Cabrero (2007).

La innovación técnica, como su nombre lo indica, transforma el sistema técnico de una organización y se relaciona con su actividad primaria: procesos y métodos de producción, mejora de productos y servicios, etc. La innovación administrativa modifica el sistema social de la organización, conformado por el sistema de relaciones entre sus integrantes. Como se aprecia en la Tabla 3, la combinación resultante de estos tipos de innovación genera cuatro categorías de organización: las del tipo D (orgánicas) que fomentan las innovaciones y por lo general son organizaciones de alta competitividad, las del tipo A (mecánicas) que no suelen innovar, y las del tipo B (administrativas burocráticas) y $\mathrm{C}$ (tecnológicas burocráticas), en las que la innovación es incierta (Cabrero et al., 2000).
Como proceso de cambio, la innovación está en función de las condiciones de las propias organizaciones, por lo que habría otra tipología basada en ello que incluye tres categorías. La primera es la innovación programada, cuyo proceso se conduce a través de la investigación y desarrollo de productos o servicios. La segunda lo representa la innovación no programada, que se produce cuando hay más recursos disponibles en la organización que los que realmente se requieren por el momento, y estos recursos se utilizan para fines innovadores. El tercer tipo lo representa la innovación angustiosa, que se genera en forma forzosa al percibirse una crisis y que requiere emprender acciones novedosas (Hall, 1996). Además, las características de la organización también fomentan la presencia de la innovación. 
Tabla 3. Tipos de innovaciones

\begin{tabular}{|c|c|c|c|}
\hline & \multicolumn{2}{|c|}{ Innovaciones administrativas } \\
\hline & & Bajo & Alto \\
\hline \multirow{4}{*}{ 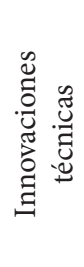 } & & Tipo A & Tipo B \\
\hline & Bajo & Mecánico & $\begin{array}{c}\text { Administrativo } \\
\text { burocrático }\end{array}$ \\
\hline & & Tipo C & Tipo D \\
\hline & Alto & $\begin{array}{l}\text { Tecnológico } \\
\text { burocrático }\end{array}$ & Orgánico \\
\hline
\end{tabular}

Fuente: De “Cambio en organizaciones gubernamentales: Innovación y complejidad”, por E. Cabrero, D. Arellano \& M. Amaya, En Reformando al gobierno. Una visión organizacional del cambio gubernamental, por D. Arellano, E. Cabrero \& A. Del Castillo, A. (coord.), 2000, $1^{\text {a }}$ ed., p. 141, México: Centro de Investigación y Docencia Económicas - M. A. Porrúa.

Los rasgos organizacionales que aparecen enseguida se vinculan con niveles altos de innovación (Hall, 1996, p. 215):

a "Gran complejidad en la capacitación profesional de los miembros de la organización. b Gran descentralización del poder.

c Poca formalización.

d Poca estratificación en las diferencias de distribución de las remuneraciones (si está presente una alta estratificación, los que tienen grandes remuneraciones probablemente resistan el cambio.

e Poco énfasis en el volumen (en oposición con la calidad) de la producción.

f Poco énfasis sobre la eficiencia en el costo de producción o de servicio.

g Un alto nivel de satisfacción con el trabajo de parte de los miembros de la organización."

De manera complementaria y con el afán de encontrar esas nuevas configuraciones estructurales, procesos y criterios de servicio en el gobierno, se pueden identificar tres niveles o ámbitos en los cuales implementar iniciativas de innovación en sus organizaciones: estructural-funcional, comportamental y relacional (Cabrero et al., 200o), cada uno con sus correspondientes objetivos y focos de transformación (ver Tabla 4).

\section{La NGP: un modelo innovador para la gestión pública}

\subsection{Características de la administración} pública iberoamericana

Sin lugar a duda los elementos fundamentales del modelo tradicional de administración pública se asocian al modelo burocrático, patentado por el célebre sociólogo alemán Max Weber y cuyos rasgos predominantes, como lo recuerda Paramés (1978), son los principios de la división del trabajo, permanencia, jerarquía e impersonalidad de las normas. En esa misma obra, este científico advertía de la crisis del modelo burocrático; parafraseando a M. Crozier, indica que este tipo de organización ha originado un evidente aislamiento de niveles y categorías jerárquicas, así como una abrumadora centralización en la toma de decisiones, lo que a su vez ha impulsado una gestión excesivamente rígida y lenta para responder oportunamente a los cambios y retos que el contexto produce. ${ }^{7}$

No obstante, se debe subrayar que con base al modelo burocrático, durante el siglo

7 El propio Crozier (1974, p. 86) añade que "el burocrático no es solamente un sistema que no se corrige en función de sus errores, sino también demasiado rígido como para adaptarse a las transformaciones que la acelerada evolución de las sociedades industriales hace cada vez más imperiosamente necesarias sin sufrir crisis". 
Tabla 4. Niveles de innovación

\begin{tabular}{|c|c|c|c|}
\hline Nivel & Objetivos & Resultados & Foco de la transformación \\
\hline $\begin{array}{l}\text { Funcional- } \\
\text { estructural }\end{array}$ & $\begin{array}{l}{ }^{*} \text { Hacer nuevas cosas de una } \\
\text { nueva manera } \\
{ }^{\star} \text { Nueva combinación de } \\
\text { recursos } \\
{ }^{\star} \text { Nuevas formas organizativas }\end{array}$ & $\begin{array}{l}\text { Transformación } \\
\text { estructural }\end{array}$ & $\begin{array}{l}{ }^{*} \text { Marco normativo } \\
{ }^{\star} \text { Nuevos procesos de trabajo } \\
{ }^{\star} \text { Reformas administrativas } \\
{ }^{\star} \text { Diseño de nuevas estructuras } \\
{ }^{\star} \text { Ampliación de grados de auto- } \\
\text { nomía }\end{array}$ \\
\hline $\begin{array}{l}\text { Comporta- } \\
\text { mental }\end{array}$ & ${ }^{*}$ Nuevas actitudes y valores & $\begin{array}{l}\text { Transformación } \\
\text { del proceso de } \\
\text { toma de deci- } \\
\text { siones }\end{array}$ & $\begin{array}{l}{ }^{*} \text { Identificación con valores } \\
{ }^{\star} \text { Mecanismos de resolución de } \\
\text { conflictos } \\
{ }^{\star} \text { Estructura de poder }\end{array}$ \\
\hline Relacional & $\begin{array}{l}{ }^{\star} \text { Nuevas formas de relación } \\
\text { organización-sociedad }\end{array}$ & $\begin{array}{l}\text { Transformación } \\
\text { institucional }\end{array}$ & $\begin{array}{l}{ }^{\star} \text { Procesos adaptativos } \\
{ }^{\star} \text { Alianzas y asociaciones con } \\
\text { agentes del entorno }\end{array}$ \\
\hline
\end{tabular}

Fuente: Elaboración propia, adaptado de Cabrero et al. (2000, p. 151).

$\mathrm{xx}$ se crearon, gestionaron y expandieron las grandes corporaciones manufactureras y de servicios, públicas y privadas, en una sociedad demandante de múltiples productos y servicios a gran escala. Asimismo y para ser justos con Weber, hay que añadir que tal como lo señala el CLAD (1998), en América Latina no se aplicaron adecuadamente varias de las ideas básicas en torno al modelo burocrático, como lo correspondiente a la profesionalización y meritocracia de los funcionarios públicos debido a prácticas patrimonialistas y clientelistas. A pesar de lo anterior, el CLAD coincide con Crozier al subrayar que este modelo no contempla algunos de los rasgos de la modernización del sector público como la eficiencia, la democratización del servicio público y la flexibilidad organizacional.

Los problemas vinculados a las agencias públicas, junto a las desventajas inherentes a su gestión presentadas en la Tabla 1, ocasionaron a su vez la presencia de rasgos no muy favorables en sus procesos administrativos $\mathrm{y}$, fundamentalmente, en su relación con su clientela: la ciudadanía. En esto coinciden importantes autores como Paramés (1978) y
Kliksberg (1989) al referirse, respectivamente, a las diversas patologías en el ámbito de la administración pública y el Estado. La Tabla 5 presenta dichos rasgos.

Por si lo anterior no fuera suficiente, la valoración de la ciudadanía acerca de los servicios y el trato ofrecidos por las instituciones del Estado tampoco resulta favorable. De acuerdo al Programa de las Naciones Unidas para el Desarrollo -PNUD (2004), en 2002 un $40 \%$ de los latinoamericanos manifestó haber acudido a una institución pública a realizar algún trámite; y de ellos, un $78 \%$ señaló haber recibido algún tipo de maltrato. ${ }^{8}$ Igualmente, la satisfacción con los servicios públicos en la región es muy baja, ya que los que ofrece el gobierno central presentan la siguiente valoración: el registro civil alcanza el 58\% de satisfacción, la educación un $54 \%$, los hospitales públicos el $47 \%$, la policía el $34 \%$ y, por último, el sistema judicial un bajo 31\%. La situación

8 A pesar que en la mayoría de los casos se trató de maltratos leves (como el hacer largas filas, trámites innecesarios, negación de información, etc.), aproximadamente una cuarta parte señaló haber sufrido maltrato grave, como el ser humillado, recibir trato irrespetuoso o recibir una invitación a dar propina. 
Tabla 5. Características de las agencias gubernamentales iberoamericanas

\begin{tabular}{|c|c|c|c|}
\hline No. & Autor & Ámbito de estudio & Patologías encontradas \\
\hline 1 & Paramés & $\begin{array}{l}\text { Administración } \\
\text { pública }\end{array}$ & $\begin{array}{l}\text { Generalizada insatisfacción de los ciudadanos } \\
\text { Deficiencias de las estructuras administrativas } \\
\text { La inadecuación de las normas jurídicas } \\
\text { Ineficiente actuación del personal: } \\
\text { - Inexperiencia, falta de capacitación, bajo espíritu de servi- } \\
\text { cio, despotismo, etc. }\end{array}$ \\
\hline 2 & Kliksberg & Estado & $\begin{array}{l}\text { Bajo nivel de articulación organizacional } \\
\text { Hiperconcentración del aparato estatal } \\
\text { Extrema rigidez } \\
\text { Ineficiencias serias en la elaboración de políticas públicas } \\
\text { Carencia de una política de personal }\end{array}$ \\
\hline
\end{tabular}

Fuente: Elaboración propia con base a Paramés (1978, pp. 484-492) y Kliksberg (1989, pp. 95-101).

no es mejor en los gobiernos municipales: la disponibilidad de áreas verdes y espacios públicos llega al 52\% de satisfacción, el servicio de recolección de basura alcanza el 50\%, los servicios municipales registran el $46 \%$, el alcantarillado el 44\%, el transporte público el $40 \%$ y los caminos y pavimentación el $41 \%$ (Corporación Latinobarómetro, 2010).

\subsection{La NGP}

Ante ese panorama, resultaba necesario emprender una cruzada multinacional que revitalizara la gestión pública. El referente para ese proyecto fueron las transformaciones que se vienen implementando desde la década de los años ochenta del siglo pasado en los sectores públicos de países desarrollados como Australia, Estados Unidos, Nueva Zelanda y Reino Unido. Estos cambios, de diversa naturaleza y magnitud, se englobaron en el modelo administrativo conocido como NGP, el cual, de acuerdo a Shand (1996), ha producido dos tipos de cambio: cambios macro y cambios micro. Los primeros incluyen cambios estructurales en el gobierno, políticas de privatización y el establecimiento de agencias autónomas. Mientras que los cambios micro, más empleados en los países de la ocDE, implican programas más efectivos (como el de mejorar las metas), operaciones más eficientes y el mejoramiento de la calidad y prestación de los servicios. Los componentes de este modelo se resumen en la Tabla 6 .

Además, la NGP aglutina un buen número de modelos, autores, filosofías y estrategias específicas. En el caso de los Estados Unidos, sobresalen las propuestas de Barzelay y de Osborne y Gaebler. ${ }^{9}$ El planteamiento de Barzelay (2000), conocido como paradigma posburocrático, reafirma que el modelo burocrático-weberiano sufrió un agotamiento y no es útil para enfrentar la nueva dinámica social y organizacional. Barzelay indica que la gestión presupuestal en el gobierno está basada en la idea del gasto y el ahorro, no en la manera más eficiente de alcanzar objetivos, y que los mecanismos de mercado deben ser incorporados para solucionar problemas de dispendio y lentitud en su actuación. La Tabla 7 integra los rasgos que, en contraste con las agencias burocráticas, deben tener las organizaciones posburocráticas para enfrentar esta nueva realidad.

9 Ambas aparecidas originalmente en 1992; en el caso de Barzelay con el nombre de "Breaking through Bureaucracy”, mientras que la de Osborne y Gaebler se identificó como "Reinventing government". 
Tabla 6. Componentes de la NGP

\begin{tabular}{|c|c|}
\hline No. & Componente \\
\hline 1 & $\begin{array}{l}\text { Enfocarse más de cerca a los resultados en términos de eficiencia, eficacia y calidad del servi- } \\
\text { cio. }\end{array}$ \\
\hline 2 & $\begin{array}{l}\text { Descentralizar las estructuras organizacionales altamente centralizadas. Además que la ges- } \\
\text { tión de servicios sea más cercana al punto de la prestación. }\end{array}$ \\
\hline 3 & Flexibilidad para explorar alternativas en la provisión directa al público. \\
\hline 4 & Nuevas políticas de manejo de personal que den mayor flexibilidad al desarrollo del staff. \\
\hline 5 & $\begin{array}{l}\text { Usar mecanismos para mejorar cuestiones como el desempeño en la concesión y crear medios } \\
\text { competitivos y de mercado dentro de las organizaciones públicas. }\end{array}$ \\
\hline 6 & $\begin{array}{l}\text { Incentivos para mejorar el desempeño que permitan a las organizaciones lograr porcentajes } \\
\text { significativos de ahorros en su administración. }\end{array}$ \\
\hline 7 & Fortalecimiento de capacidades estratégicas del gobierno. \\
\hline 8 & Mayor responsabilidad y transparencia para reportar los resultados de gestión. \\
\hline 9 & Un enfoque al cliente o al consumidor. \\
\hline
\end{tabular}

Fuente: Elaboración propia con base en Shand (1996, pp. 82-88).

Tabla 7. Características de las organizaciones posburocráticas

\begin{tabular}{|c|c|c|}
\hline No. & $\begin{array}{l}\text { Características de una dependencia } \\
\text { burocrática }\end{array}$ & $\begin{array}{l}\text { Características de una dependencia } \\
\text { posburocrática o impulsada por el cliente }\end{array}$ \\
\hline 1 & $\begin{array}{l}\text { Se enfoca en sus propias necesidades y pers- } \\
\text { pectivas }\end{array}$ & $\begin{array}{l}\text { Se enfoca hacia las necesidades y perspectivas del } \\
\text { cliente }\end{array}$ \\
\hline 2 & $\begin{array}{l}\text { Se enfoca en las funciones y las responsabilida- } \\
\text { des de sus partes }\end{array}$ & $\begin{array}{l}\text { Se enfoca en ayudar a que toda la organización funcio- } \\
\text { ne como equipo }\end{array}$ \\
\hline 3 & $\begin{array}{l}\text { Se define tanto por la cantidad de recursos que } \\
\text { controla como por las tareas que desempeña }\end{array}$ & $\begin{array}{l}\text { Se define por los resultados que logra en beneficio de } \\
\text { sus clientes }\end{array}$ \\
\hline 4 & Controla los costos & Crea un valor neto de costo \\
\hline 5 & Se aferra a la rutina & $\begin{array}{l}\text { Modifica sus operaciones en respuesta a las demandas } \\
\text { cambiantes de sus servicios }\end{array}$ \\
\hline 6 & Lucha por ganar terreno & Compite por obtener más negocio \\
\hline 7 & Insiste en seguir los procedimientos normales & $\begin{array}{l}\text { Introduce la opción en sus sistemas operativos, cuando } \\
\text { al hacerlo cumple un propósito }\end{array}$ \\
\hline 8 & Anuncia políticas y planes & $\begin{array}{l}\text { Emprende con sus clientes una comunicación en am- } \\
\text { bos sentidos, con objeto de evaluar y revisar su estrate- } \\
\text { gia operativa }\end{array}$ \\
\hline 9 & Separa el trabajo de pensar del trabajo de hacer & $\begin{array}{l}\text { Otorga la autoridad a los empleados operativos de emi- } \\
\text { tir juicios sobre el modo de mejorar tanto el servicio al } \\
\text { cliente como su valor }\end{array}$ \\
\hline
\end{tabular}

Fuente: Elaboración propia con base en Barzelay (2000, p. 47).

Casi de manera simultánea, se presentó la propuesta de Osborne \& Gaebler (1994), conocida como la reinvención del gobierno, en la cual el gobierno es visto como socio del sector privado para enfrentar los problemas nacionales prioritarios; es decir, para llegar a ser ese nuevo socio, el gobierno necesita reinventarse. Basados en las ideas de Osborne y Gaebler, Jones \& Thompson (1999) proponen un modelo de gestión conocido 
como 5R's, mismo que constituye una plataforma para la innovación en la gestión pública al incorporar los siguientes conceptos: reestructuración, reingeniería, reinvención, realineación y reconceptualización. El fundamento de estos conceptos, cuya utilidad para el ámbito público es demostrada por Fernández, Delgado, Ortega \& Caldera (2007), se explica en la Tabla 8.

En suma, la NGP representa una alternativa a una gestión pública tradicional apegada a funciones rutinarias y temerosas de experimentar con mecanismos innovadores; asimismo, este modelo propone construir organizaciones orgánicas a partir de las organizaciones mecánicas o burocráticas existentes. Esto implica conseguir innovaciones en los niveles funcional-estructural (por medio de programas de calidad, reingeniería y reestructuración), comportamental (a través de nuevas políticas de personal y una nueva concepción del servicio público) y relacional (enfoque en el cliente-ciudadano y asociación con empresas privadas), herramientas todas ellas que incorpora la NGP.

\subsection{La NGP en la administración pública de Iberoamérica}

Como se señaló en la introducción de este trabajo y como lo constatan un buen número de publicaciones (CLAD, 2010; García, 2004; Guillén, González \& Rojo, 2007; Guillén, López \& Rojo, 2006; Guillén \& Rojo, 2005), desde hace varios años se vienen implementando en los gobiernos iberoamericanos programas de mejoramiento, modernización, reingeniería o reinvención, los cuales se alinean en forma clara a los principios de la NGP.

Tabla 8. Fundamentos del modelo 5R's

\begin{tabular}{|c|c|c|}
\hline No. & Componente & Fundamento \\
\hline 1 & Reestructuración & $\begin{array}{l}\text { Tal como lo han señalado Crozier (1974), Paramés (1978), Kliksberg (1989), Shand } \\
\text { (1996) y Barzelay (2000), el gobierno requiere trabajar con estructuras más ágiles } \\
\text { y funcionales, más planas (con menos niveles jerárquicos) y diseñadas para hacer } \\
\text { más eficientes los procesos. }\end{array}$ \\
\hline 2 & Reingeniería & $\begin{array}{l}\text { Hammer \& Champy (1993) indican que la reingeniería constituye la mejora ra- } \\
\text { dical de los procesos de trabajo de una organización para obtener elevados be- } \\
\text { neficios en costo, servicio y rapidez. Esa idea habría que llevarla al interior de las } \\
\text { administraciones públicas, plagadas de trámites lentos y engorrosos; el trabajo de } \\
\text { Torres (2007) demuestra que sí es posible su implementación en ellas. }\end{array}$ \\
\hline 3 & Reinvención & $\begin{array}{l}\text { La reinvención significa la transformación fundamental de los organismos y } \\
\text { sistemas públicos para que produzcan mejoras espectaculares en su eficacia, su } \\
\text { eficiencia, su adaptabilidad y su capacidad para innovar. Esta transformación se } \\
\text { logra cambiando su propósito, sus incentivos, su responsabilidad, su estructura } \\
\text { de poder y su cultura (Osborne \& Plastrik, 1998). }\end{array}$ \\
\hline 4 & Realineación & $\begin{array}{l}\text { Se propone alinear o hacer que coincidan, que haya congruencia entre la estruc- } \\
\text { tura y la estrategia de la organización para poder alcanzar sus fines, a la vez que } \\
\text { se motiva y responsabiliza al personal. Cabe resaltar que la importancia de tal } \\
\text { alineación de variables fue antes estudiada por autores ahora clásicos de la Teoría } \\
\text { de la Organización como Chandler (1962). }\end{array}$ \\
\hline 5 & Reconceptualización & $\begin{array}{l}\text { Plantea fomentar la innovación, la creatividad y el autoaprendizaje en el sector } \\
\text { público con el fin de identificar nuevos mercados, clientes, productos, así como } \\
\text { formas de organización y mecanismos de atención. Ideas que guardan amplia } \\
\text { relación con la propuesta sobre innovación y espíritu emprendedor de Koontz, } \\
\text { Weihrich \& Cannice (2008). }\end{array}$ \\
\hline
\end{tabular}

Fuente: Elaboración propia a partir de Jones \& Thompson (1999). 
El CLAD ha sido un destacado promotor de este tipo de iniciativas, particularmente desde la creación de un manifiesto que se convirtió en un referente en la región: Una nueva gestión pública para América Latina (CLAD, 1998). Posteriormente y hasta estos momentos, esta iniciativa ha seguido generando documentos inspirados en la NGP, los que a la vez han fomentado su aplicación en los gobiernos iberoamericanos. Por su vinculación directa con la teoría de la innovación, en este trabajo se revisarán, de acuerdo a la información de la Tabla 9, tres de ellos, firmados por la mayoría de los gobiernos de la región: las Carta Iberoamericanas de la Función Pública (CLAD, 2003), de Gobierno Electrónico (CLAD, 2007) y de Calidad en la Gestión Pública (CLAD, 2008).

Como se puede observar, en términos generales estos documentos recogen linealmente las ideas fundamentales de los autores impulsores de la NGP (lo que les convierte en verdaderas guías para la innovación): una gestión pública más eficazy eficiente, más flexible, preocupada por servir a la ciudadanía, que ofrezca servicios de mayor calidad y cuyos rendimientos sean evaluados. En lo particular, la CIFP promueve una gestión pública basada en los resultados, la innovación y el aprendizaje, particularmente en lo que respecta a la gestión del personal. Mientras que la CIGE se preocupa por la mejora continua de los procesos y su constante innovación con base en las TIC. Por su parte, la CICGP incorpora una propuesta básica de la NGP: el aprendizaje y la innovación como instrumentos vitales en la estrategia de mejoramiento de la calidad.

\section{Análisis de tres experiencias innovado- ras en gobiernos locales}

En esta sección se presentan tres estudios de caso que constatan que, a pesar de que tradicionalmente no se han distinguido por tener altos niveles de desempeño, la implementa- ción de proyectos innovadores exitosos, guiados por ideas de NGP, es una cabal posibilidad en los gobiernos locales iberoamericanos. Se estudiarán sucintamente las experiencias del Servicio de Atención al Ciudadano (SAC), la Ciudad Rural Sustentable (CRs) de Nuevo Juan de Grijalva y de Eco-Impulso.

\subsection{Servicio de Atención al Ciudadano $(S A C)^{10}$}

Este proyecto corre a cargo de la Secretaría de Administración del gobierno del estado de Bahía (Brasil) e inicia en 1995 impulsado por la imagen que tenía este gobierno de brindar un servicio público ineficiente. Para transformar esa reputación se creó el SAC, que constituye una transformación radical en la manera de ofrecer servicios públicos y que se puede definir como un shopping de servicios públicos, en el que agencias del gobierno, federal, provincial y municipal, junto a empresas concesionarias privadas, ofertan los servicios públicos más importantes con parámetros de excelencia. Los centros SAC se ubican en lugares convenientes para la ciudadanía, además de shopping centers, estaciones de transporte público y colonias de bajo ingreso; asimismo cuentan con dos unidades móviles para llevar a las comunidades más alejadas y marginadas los servicios públicos más elementales.

Con el sAC se logró que, en un mismo lugar, 32 instituciones de los tres niveles de gobierno ofrecieran seiscientos tipos de servicios a los ciudadanos con economías de tiempo y costo, además de confort en las instalaciones, un trato respetuoso y servicios de apoyo como fotocopiado, puesto bancario y fotografías. En cada centro hay un gerente

10 A continuación se presenta un resumen adaptado a partir del documento del SIPAL -Sistema Regional de Información sobre Buenas Prácticas de Gestión Pública en América Latina y El Caribe (2006). Más información en: http://www.sac.ba.gov.br y http://www.clad.org/siare_isis/ innotend/gobelec/ccbrasil.html 
Innovación en los gobiernos locales en Iberoamérica. Posibilidades a partir de la nueva gerencia pública

Tabla 9. Documentos signados por gobiernos iberoamericanos inspirados en la NGP

\begin{tabular}{|c|c|c|c|}
\hline & $\begin{array}{l}\text { Carta Iberoamericana de la } \\
\text { Función Pública (CIFP) }\end{array}$ & $\begin{array}{l}\text { Carta Iberoamericana de Go- } \\
\text { bierno Electrónico (CIGE) }\end{array}$ & $\begin{array}{l}\text { Carta Iberoamericana } \\
\text { de Calidad en la Gestión } \\
\text { Pública (CICGP) }\end{array}$ \\
\hline Meta & $\begin{array}{l}\text { Definir las bases que configu- } \\
\text { ran un sistema eficaz y profe- } \\
\text { sional de función pública }\end{array}$ & $\begin{array}{l}\text { Acercar los gobiernos a la } \\
\text { ciudadanía al facilitar la } \\
\text { comunicación y la relación } \\
\text { vía las TIC }\end{array}$ & $\begin{array}{l}\text { Promover un marco } \\
\text { común en Iberoamérica } \\
\text { sobre la calidad en la } \\
\text { gestión pública }\end{array}$ \\
\hline \multirow[t]{7}{*}{ Criterios } & Prioridad de las personas & Calidad de los servicios & Servicio a la ciudadanía \\
\hline & La profesionalidad & Eficiencia, eficacia & Gestión para resultados \\
\hline & Flexibilidad & Transparencia & Compromiso social \\
\hline & Responsabilidad & $\begin{array}{l}\text { Sociedad de la información y } \\
\text { del conocimiento }\end{array}$ & Responsabilidad directiva \\
\hline & Principios éticos & \multirow[t]{3}{*}{ Gobernabilidad democrática } & Gestión por procesos \\
\hline & Protagonismo (liderazgo) & & $\begin{array}{l}\text { Participación de emplea- } \\
\text { dos }\end{array}$ \\
\hline & $\begin{array}{l}\text { Igualdad de género, la inclu- } \\
\text { sión y no discriminación }\end{array}$ & & Cooperación \\
\hline \multirow{5}{*}{$\begin{array}{l}\text { Principios } \\
\text { básicos }\end{array}$} & Igualdad & Igualdad & Servicio público \\
\hline & Mérito, desempeño & Legalidad & Participación ciudadana \\
\hline & Eficacia, eficiencia & Conservación & Acceso universal \\
\hline & Transparencia, objetividad & Responsabilidad & Eficacia, economía \\
\hline & Sometimiento a la ley & Adecuación tecnológica & Mejora Continua \\
\hline \multirow[t]{7}{*}{$\begin{array}{l}\text { Algunos instru- } \\
\text { mentos }\end{array}$} & $\begin{array}{l}\text { Planificación de recursos } \\
\text { humanos }\end{array}$ & Los ciudadanos podrán: & Dirección estratégica \\
\hline & Organización del trabajo & $\begin{array}{l}\text { Enviar vía electrónica escritos } \\
\text { y quejas }\end{array}$ & Opinión ciudadana \\
\hline & Evaluación del rendimiento & $\begin{array}{l}\text { Realizar pagos, liquidar im- } \\
\text { puestos }\end{array}$ & Modelos de excelencia \\
\hline & Función directiva profesional & Recibir notificaciones & Premios a la calidad \\
\hline & \multirow[t]{3}{*}{ Cambio cultural } & \multirow[t]{3}{*}{ Acceder a información } & Equipos de mejora \\
\hline & & & Cartas compromiso \\
\hline & & & Medición y evaluación \\
\hline \multirow[t]{2}{*}{$\begin{array}{l}\text { Referencia a la } \\
\text { innovación }\end{array}$} & $\begin{array}{l}\text { En las políticas y prácticas de } \\
\text { gestión de las personas }\end{array}$ & \multirow{2}{*}{$\begin{array}{l}\text { Los Estados deberán implan- } \\
\text { tar nuevos modelos de gestión } \\
\text { en la administración pública } \\
\text { que fomenten la mejora } \\
\text { continua de los procesos y la } \\
\text { constante innovación }\end{array}$} & $\begin{array}{l}\text { La administración deberá } \\
\text { establecer un proceso de } \\
\text { aprendizaje, innovación y } \\
\text { mejora de la calidad }\end{array}$ \\
\hline & $\begin{array}{l}\text { Suplir rutinas y procedimien- } \\
\text { tos por enfoques orientados a } \\
\text { la producción de resultados, la } \\
\text { innovación y el aprendizaje }\end{array}$ & & $\begin{array}{l}\text { Los ciudadanos podrán } \\
\text { participar en el diseño e } \\
\text { innovación del servicio }\end{array}$ \\
\hline
\end{tabular}

Fuente: Elaboración propia con base en el CLAD (2003, 2007, 2008). 
que pertenece al gobierno de Bahía y a él le reporta el supervisor que cada agencia gubernamental tiene en ese lugar. Los recursos humanos que prestan sus servicios en las agencias son funcionarios públicos pertenecientes a las mismas agencias o a empresas privadas que prestan servicio en el SAC (outsourcing).

El sAc cuenta con un total de 25 centros fijos (ocho en Salvador, capital de Bahía, y diecisiete en el interior) y dos puestos móviles, que durante tres o cuatro días recorren los 417 municipios de Bahía ofreciendo los servicios de acta de nacimiento, cédula de identidad y certificado de no antecedentes penales. Entre los resultados más significativos de la experiencia se tienen: hasta 2005 se habían realizado 78 millones de atenciones; los tiempos de espera para ser atendido en el sAc se han abatido significativamente, por ejemplo, un pasaporte puede obtenerse en un tiempo promedio de ocho minutos; además, la encuesta 2005 indicó una tasa de satisfacción de $93,9 \%$ con los servicios ofrecidos entre los usuarios del sac. Todo ello ha posibilitado que el sAc haya sido galardonado con el Premio al Servicio Público de la Organización de las Naciones Unidas en la categoría "Mejora en los Resultados de los Servicios Públicos" en el 2004, y sea reconocido como modelo de referencia en la prestación de servicios administrativos al público.

\subsection{Ciudad Rural Sustentable (CRS) Nuevo Juan del Grijalva (México)"11}

$\mathrm{El} 4$ de noviembre de 2007, debido a las fuertes lluvias, se desgajó un cerro sobre el Río Grijalva, lo que provocó una inmensa ola que destruyó la localidad Juan del Grijalva en el Estado de Chiapas (México). A raíz de esto, el gobierno de Chiapas se propuso construir

11 Enseguida se leerá una síntesis propia hecha a partir del estudio del Instituto de Población y Ciudades Rurales del Gobierno del Estado de Chiapas (2011). la primera CRS y con ello brindar la oportunidad a las familias damnificadas de emprender una nueva vida de manera digna. Esa primera CRs es el Nuevo Juan del Grijalva; en donde, en su edificación (que implicó la adquisición de un predio de ochenta hectáreas a $7 \mathrm{~km}$ de Ostuacán, la cabecera municipal), se han integrado los cinco componentes básicos del proyecto CRs: vivienda; desarrollo social; económico, productivo y de servicios; sustentabilidad ambiental; y legalidad y gobierno. A continuación se muestran sus resultados.

Vivienda. Se han construido 410 casas de $60 \mathrm{~m}^{2}$ con paredes de adoblock (elaborado combinando cemento, arena y tierra de la región), que cuentan con estufas ecológicas, predios de $300 \mathrm{~m} 2$, traspatios productivos de $240 \mathrm{~m} 2$ (para la crianza de animales, hortalizas y frutales), agua potable, electricidad, drenaje sanitario y pluvial y alumbrado público. La CRS cuenta con planta de tratamiento de aguas residuales, red de energía eléctrica con cableado subterráneo, sistema de alumbrado público de energía solar, terminal de transporte público, templos religiosos, jardines comunes y canchas deportivas.

Desarrollo Social. La CRS cuenta con un centro de salud que brinda 142 servicios, un consultorio de medicina general y otro de odontología; un centro de educación básica (СЕBECH) que durante el ciclo escolar 20092010 atendió a 470 alumnos; un centro de asistencia infantil comunitario con servicio de salud y alimentación para apoyar a madres trabajadoras; un centro de desarrollo comunitario; lo que se complementa con una torre de comunicaciones que posibilita los servicios de internet y telefonía celular y que cuenta con sala audiovisual, sala de cómputo, biblioteca, además de albergar una radio comunitaria.

Económico, productivo y de servicios. Se construyó un corredor comercial, un parque microindustrial y diez distintas instalacio- 
nes comerciales y de servicios; en ellos se encuentran operando 48 microempresas, destacando una fábrica de muebles, otra de blocks, una herrería, una tortillería, un molino, una posada rural, cinco tiendas de abarrotes y una panadería. Asimismo, se han impulsado varios proyectos agroindustriales: tres granjas de aves de postura para la producción de huevo administradas por mujeres, una planta procesadora de lácteos, ocho invernaderos de tomate, chile habanero y otras hortalizas, una planta empacadora hortofrutícola y una planta fermentadora y secadora de cacao.

Sustentabilidad Ambiental. Se reforestaron las cincuenta hectáreas del polígono urbano, incluyendo un terreno como reserva ecológica; también se reforestaron las treinta hectáreas del polígono productivo, en donde se sembraron especies frutales y maderables para su futuro aprovechamiento. La CRS dispone del servicio de recolección de basura por medio de camiones que clasifican los residuos orgánicos e inorgánicos.

Legalidad y gobierno. La ciudad cuenta con una Delegación Municipal, una Comandancia Municipal y una Agencia del Ministerio Público, las cuales están dotadas con suficiente infraestructura y equipamiento necesario para su operación.

\subsection{Eco-Impulso ${ }^{12}$}

Esta experiencia es promovida por la Dirección de Ecología del gobierno municipal de Tepatitlán de Morelos, Jalisco (México), debido a la necesidad de movilizar a los ciudadanos en la mejora del medio ambiente y generar una cultura ecológica. Para tal fin, este programa incluye actualmente cuatro subprogramas: 1. Reciclaje Ecológico Escolar,

12 El siguiente es un extracto libre elaborado con base en Díaz \& Bazdresch (2005), disponible también en http:// premiomunicipal.org.mx/p20o9/pa_descargar_doc. php?idRegistro $=3477 \&$ tipo $=$ doc
2. "Creciendo Juntos", 3. "Salvemos al Río" y 4. "Eco bonos".

El Reciclaje Ecológico Escolar inicia en 1994 y consiste en que niños de escuelas de educación básica, ubicadas en zonas de bajos ingresos, lleven a sus colegios residuos reciclables, los cuales semanalmente se canalizan al Centro Municipal de Acopio (construido en 1992). Al final del año escolar, se entrega un reconocimiento y una cantidad en efectivo (peso por peso) a las escuelas en que se hayan recolectado más residuos.

La iniciativa Creciendo Juntos invita a los niños de educación básica al vivero municipal para recibir pláticas sobre cuidado de árboles, además de que aprenden a sembrar sus semillas y se les pide que escojan una que habrán de cuidar para que crezca junto con ellos. De manera complementaria se ofrece a las escuelas y colonias la siembra de árboles pequeños, cultivados en el mismo vivero municipal, para que sean plantados en banquetas, patios y jardines domésticos con el compromiso de parte de los niños, sus profesores y padres de familia de cuidarlos hasta su crecimiento.

El programa Salvemos al Río es un plan de gobierno provocado por el desbordamiento del Río Tepatitlán en 2003, lo que ocasionó cuantiosos daños en construcciones cercanas al río. Entre el gobierno y los ciudadanos han contribuido al desazolve del cauce, a ensancharlo, a limpiarlo y retirar árboles obstructores, a reparar los destrozos, a retirar drenajes clandestinos y descargas industriales prohibidas, a acondicionar áreas de recreo e identificar zonas de peligro potencial.

Finalmente, los Eco-bonos son un subprograma que se fundamenta en una campaña de pago en forma de vales canjeables por mercancías en comercios locales a los ciudadanos que llevan residuos sólidos reciclables al Centro de Acopio. 
Entre los logros de Eco-Impulso figura el hecho que el programa de reciclaje escolar se ha sostenido por más de diez años con el apoyo de 45 escuelas, padres de familia, ciudadanos y empresarios, además que ya cuenta con un Reglamento Municipal de Reciclaje (en el que se establece la obligación de los vecinos de separar los residuos sólidos susceptibles de ser reciclados). Como dato, en el periodo 2001-2003 se recolectaron 629 toneladas de residuos reciclables en el Centro de Acopio Municipal, 212 en las escuelas, 16 en el Cambalache Ecológico (mismas que fueron cambiadas por despensas en las zonas más pobres) y 11 en el Cambalache Escolar (que se canjearon por útiles escolares en colegios de zonas deprimidas). Otro logro es la protección de las especies arbóreas en peligro de extinción, acción que permite mantener varias decenas de miles de árboles pequeños en el vivero. Un tercer logro lo representa la protección ecológica del río, que implica a la vez la protección civil y del patrimonio de la ciudad. Todo esto llevó a que la experiencia recibiera el "Premio Gobierno y Gestión Local 2004", que entrega anualmente el CIDE en México.

La Tabla 10 presenta el análisis comparativo de los tres casos respecto a sus objetivos, estrategias y resultados, el cual indica, sin duda alguna, el empleo de acciones innovadoras, tanto de carácter administrativo como técnico, y que han impactado, en mayor o menor medida, los niveles funcionalestructural, comportamental y relacional de las organizaciones que los desarrollaron. Asimismo, es evidente que el propósito y naturaleza de la mayoría de estas acciones, entre las que se encuentran la búsqueda de una mayor eficiencia y calidad en la prestación de servicios, reinvención en la gestión, reingeniería de procesos, reconceptualización de la administración pública, una mejor vinculación con el sector empresarial y cambio cultural en la organización, se apegan claramente a los arquetipos de la NGP.

\section{Conclusiones}

Después del recorrido de carácter teórico y documental acerca de la necesidad de innovar las prácticas de gestión en los gobiernos locales de Iberoamérica, así como del análisis sucinto de tres experiencias que se alinean y responden a esa exigencia con la finalidad de ofrecer servicios renovados y una nueva relación con la ciudadanía, se pueden señalar las siguientes conclusiones:

En primer lugar, en función del agotamiento del modelo burocrático, parece observarse en muchos de estos gobiernos una mutación respecto a las formas en que tratan de cumplir sus misiones y objetivos, y que los estaría acercando a modelos de nueva gerencia pública. Esta investigación evidencia en tal sentido, la presencia de una gradual mudanza en el perfil de las organizaciones que integran los sectores públicos de América Latina, pasando de ser organizaciones burocráticas y mecánicas a convertirse, gradualmente y en función de sus innovaciones administrativas y técnicas, en organizaciones mucho más orgánicas y mejor valoradas por la ciudadanía.

Como complemento del punto anterior es posible afirmar que, con base en las experiencias en el SAC, la CRS Nuevo Juan del Grijalva y Eco-Impulso, las posibilidades para la innovación de los gobiernos municipales de la región son amplias. Para llevar a cabo proyectos de transformación e innovación exitosos, será necesario que las agencias estatales, en función del diagnóstico presentado respecto a la valoración de sus servicios por parte de la sociedad, incorporen en la mayoría de los casos los tres niveles de innovación revisados. Esto es así porque es menester cambiar estructuras y procesos obsoletos, generar nuevos valores y 
Tabla 10. Comparación de las experiencias presentadas

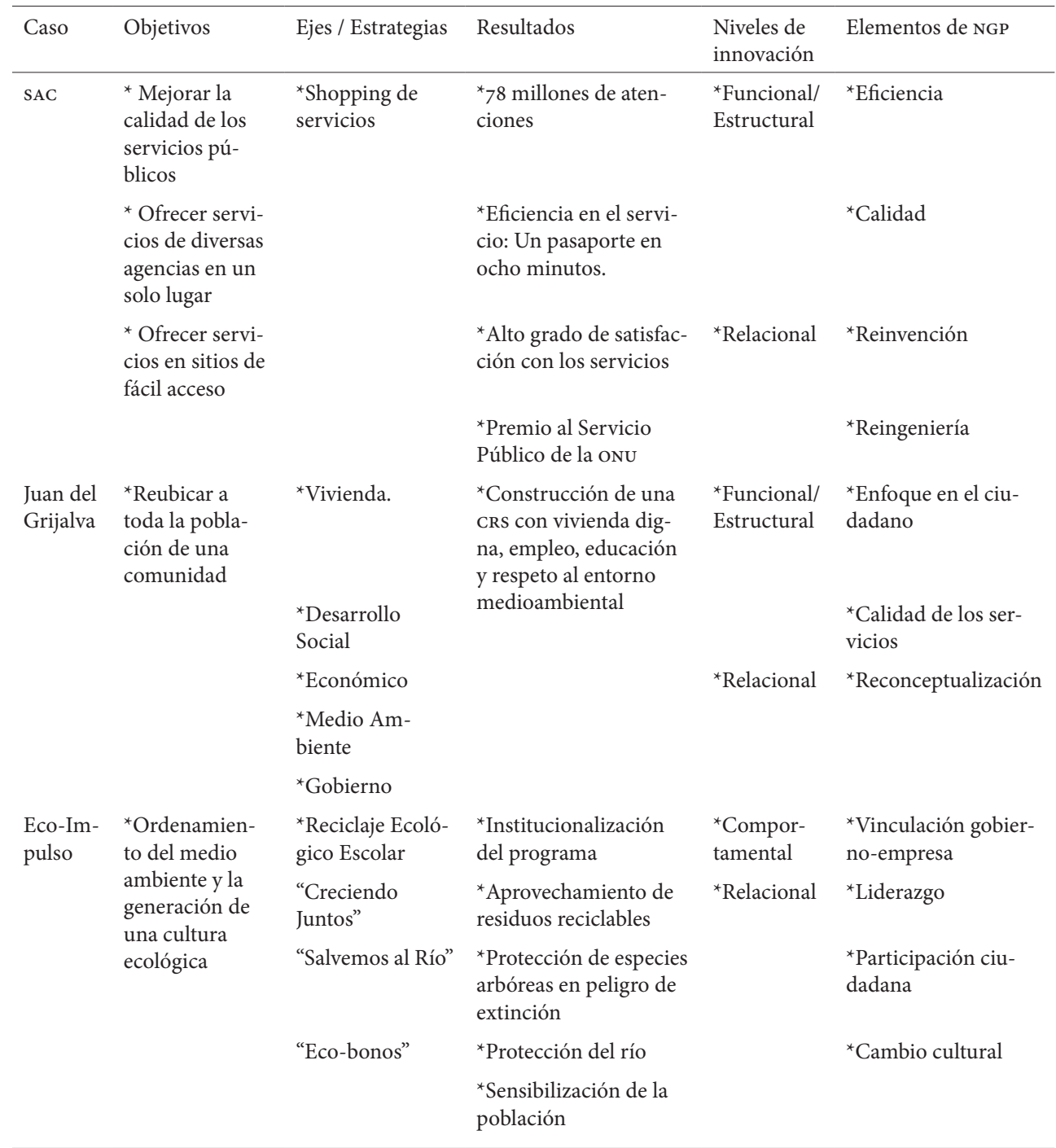

Fuente: Elaboración propia.

comportamientos (lo que se ha llamado una nueva cultura de servicio público asociada a principios éticos), así como inducir nuevas formas de vinculación con ciudadanos, grupos sociales y empresariales.

Haciendo referencia precisamente a la innovación relacional, esta investigación confirma que la nueva forma de concebir el ejercicio de gobierno sitúa al ciudadano, visto como el cliente del aparato estatal, en el centro de la acción de todo el sector. Lo anterior implica no solamente ofrecer servicios de alta calidad y eficiencia a la ciudadanía, sino que tal como se apreció en los estudios de caso revisados, los propios ciudadanos junto con las empresas deben asociarse con el gobierno en la gestión de servicios, políticas y programas públicos. 
El trabajo plantea asimismo que las ideas creativas y de cambio organizacional de los casos presentados se apegan en su mayoría al tipo de innovación angustiosa, ya que escenarios de crisis (de calidad en los servicios, administrativa $\mathrm{u}$ originada por desastres naturales) motivaron la implementación de amplias y exitosas transformaciones. Sin embargo, es recomendable generar procesos de innovación programada que hagan una adecuada lectura de las transformaciones sociales, tecnológicas, económicas y políticas que se suscitan.

La investigación también mostró que hay una clara correspondencia entre la teoría sobre la innovación y la que sustenta a la NGP. Lo que ésta propone al final del día es emprender iniciativas innovadoras que ayuden a transformar la gestión pública, en el sentido que se explicó antes y con un gran nivel de profundidad al incorporar, entre otros aspectos, programas de reingeniería y reinvención del gobierno. Igualmente, tomando como referencia las experiencias estudiadas, se corroboró la hipótesis de que la argumentación que soporta el paradigma de la NGP está sirviendo como modelo para conducir múltiples programas de reforma en los gobiernos latinoamericanos. Se debe advertir que las ideas de corte gerencial, agrupadas en la NGP, pueden ser muy útiles si se utilizan como simples medios o instrumentos, y con la correspondiente adecuación al contexto particular de que se trate, en el gran proyecto de transformación del gobierno local.

Es lógico indicar que uno de los factores que explican la diseminación de las ideas acerca de la NGP en los sectores públicos de Iberoamérica lo constituye el trabajo que instituciones como el CLAD vienen desarrollando en tal sentido desde hace algún tiempo. Al respecto, los documentos que esta institución ha elaborado con la aproba- ción de los gobiernos de la región, en torno a la función y gerencia pública, al gobierno electrónico, a la calidad en la administración pública y a la participación ciudadana, promovidos a través de diversos foros y mecanismos, tienen una valía inestimable.

Es indudable que el reto de reformar los gobiernos locales latinoamericanos debe partir del reconocimiento de sus múltiples carencias y retos en materia reglamentaria, presupuestal, operativa, administrativa y de recursos humanos. Eso implica poner en la práctica dos ideas: primero, es necesario desarrollar proyectos reformadores de manera amplia, abarcando toda la red de organizaciones que conforman a los gobiernos, en los que el empleo de ideas de NGP, si el caso es procedente, puede representar un herramental valioso. Segundo, en ese afán modernizador y partiendo de la realidad que experimentan estos gobiernos, los objetivos de tales programas deben ser razonables y realistas.

Lo anterior lleva a reconocer otra necesidad. Dado que las teorías del desarrollo ubican a la administración pública como uno de los factores vitales para generar desarrollo endógeno, es fundamental profesionalizar la gestión de recursos humanos, financiera, operativa y estratégica de los gobiernos locales. Eso sin duda también impactaría favorablemente en la calidad de vida de los ciudadanos y la sociedad iberoamericana lo requiere.

\section{Referencias bibliográficas}

Agranoff, R. (1997). Las relaciones y la gestión intergubernamentales. En R. Bañón \& E. Carrillo (comps.), La nueva administración pública (pp. 125-170). Madrid: Alianza. 
Allison, G.T. (1999). La gestión pública y la

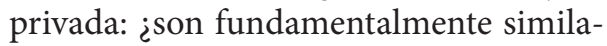
res en todos los aspectos no importantes? En J.M. Shafritz \& A.C. Hyde (Eds.), Clásicos de la administración pública (pp. 746-779). México: Fondo de Cultura Económica.

Arellano, D. \& Cabrero, E. (2000). Introducción. En D. Arellano, E. Cabrero \& A. Del Castillo, A. (coord.), Reformando al gobierno. Una visión organizacional del cambio gubernamental ( $1^{\text {a }}$ ed., pp. 5-18). México: Centro de Investigación y Docencia Económicas - M. A. Porrúa.

Banco Interamericano de Desarrollo -BID \& Centro Latinoamericano de Administración para el Desarrollo -CLAD. (2007). Modelo Abierto de Gestión Para Resultados en el Sector Público. Documentos Debate del CLAD: Estado, Administración Pública $y$ Sociedad. Caracas: CLAD.

Barzelay, M. (2000). Atravesando la burocracia. Una nueva perspectiva de la administración pública ( $1^{\mathrm{a}}$ ed.). México: Fondo de Cultura Económica.

Cabrero, E. (2007). Políticas de modernización de la administración municipal. Viejas y nuevas estrategias para transformar los gobiernos locales. En Políticas públicas municipales. Una agenda en construcción (1ra. ed.). México: Centro de Investigación y Docencia Económicas.

Cabrero, E., Arellano, D. \& Amaya, M. (2000). Cambio en organizaciones gubernamentales: Innovación y complejidad. En D. Arellano, E. Cabrero \& A. Del Castillo, A. (coord.), Reformando al gobierno. Una visión organizacional del cambio gubernamental ( $1^{a}$ ed., pp. 137168). México: Centro de Investigación y Docencia Económicas - M. A. Porrúa.

Centro Latinoamericano de Administración para el Desarrollo - CLAD. (1998). Una nueva gestión pública para América
Latina. Recuperado el 21 de junio de 2011, de http://www.clad.org/documentos/ declaraciones/una-nueva-gestionpublica-para-america-latina/view

Centro Latinoamericano de Administración para el Desarrollo -CLAD. (2003). Carta Iberoamericana de la Función Pública. Recuperado el 21 de junio de 2011, de http://www.clad.org/documentos/declaraciones/cartaibero.pdf/view

Centro Latinoamericano de Administración para el Desarrollo -CLAD. (2007). Carta Iberoamericana de Gobierno Electrónico. Recuperado el 21 de junio de 2011, de http://www.clad.org/documentos/declaraciones/cartagobelec.pdf/view

Centro Latinoamericano de Administración para el Desarrollo -CLAD. (2008). Carta Iberoamericana de Calidad en la Gestión Pública. Recuperado el 21 de junio de 2011, de http://www.clad.org/documentos/declaraciones/carta-iberoamericanade-calidad-en-la-gestion-publica/view

Centro Latinoamericano de Administración para el Desarrollo -CLAD. (2010). Gestión Pública Iberoamericana para el Siglo XXI. Recuperado el 21 de junio de 2011, de http://www.clad.org/documentos/ declaraciones/gestion-publicaiberoamericana-para-el-siglo-xxi/view

Chandler, A. (1962). Strategy and structure. Cambridge, uk: The MIT Press.

Corporación Latinobarómetro. (2010). Informe Latinobarómetro. Recuperado el 8 de junio de 2011, de http:// www.latinobarometro.org/latino/ LATContenidos.jsp

Crozier, M. (1974). El fenómeno burocrático. Buenos Aires: Amorrortu.

Díaz, G. \& Bazdresch, M. (2005). Eco-Impulso. Tepatitlán de Morelos, Jalisco. En T. Guillén \& P. Rojo (coord.), Gobernar con calidad y para el desarrollo. Experiencias de innovación en los municipios mexicanos. 
Premio Gobierno y Gestión Local 2004 (pp. 49-63). México: Centro de Investigación y Docencia Económicas.

Fernández, N., Delgado, F., Ortega, E. \& Caldera, E. (2007). SERBiLuz: Transitando hacia un modelo gerencial innovador. Revista Venezolana de Gerencia, 12(37), 99-112.

García, R. (coord.) (2004). Gestión local creativa: Experiencias innovadoras en México. Premio Gobierno y Gestión Local 2003. México: Centro de Investigación y Docencia Económicas.

Guillén, T., González, L. \& Rojo, P. (coord.) (2007). Gobierno de proximidad. La capacidad y el ingenio de la gestión local mexicana. Premio Gobierno y Gestión Local 2006. México: Centro de Investigación y Docencia Económicas.

Guillén, T., López, P. \& Rojo, P. (coord.) (2006). Municipio y buen gobierno: Experiencias del ímpetu local en México. Premio Gobierno y Gestión Local 2005. México: Centro de Investigación y Docencia Económicas.

Guillén, T. \& Rojo, P. (coord.). (2005). Gobernar con calidad y para el desarrollo. Experiencias de innovación en los municipios mexicanos. Premio Gobierno y Gestión Local 2004. México: Centro de Investigación y Docencia Económicas.

Hall, R. (1996). Organizaciones. Estructuras, procesos y resultados ( $6^{\mathrm{a}}$ ed.). México: Prentice Hall Hispanoamericana.

Hammer, M. \& Champy, J. (1993). Reengineering the corporation: A manifesto for business revolution (1a ed.). New York, NY: Harper Collins.

Hernández, R., Fernández, C. \& Baptista, P. (2007). Metodología de la investigación ( $4^{\mathrm{a}}$ ed.). México: McGraw-Hill Interamericana.

Instituto de Población y Ciudades Rurales del Gobierno del Estado de Chiapas.
(2011). Ciudades Rurales Sustentables: Nuevo Juan del Grijalva. Recuperado el 9 de julio de 2011, de http://www. ciudadesrurales.chiapas.gob.mx/nuevojuan-del-grijalva

Jones, G. \& George, J. (2006). Administración contemporánea (4ta. ed.). México: McGraw-Hill Interamericana.

Jones, L.R. \& Thompson, F. (1999). Un modelo para la nueva gerencia pública: lecciones de la reforma de los sectores público y privado. Recuperado el 21 de junio de 2011, de http://www.clad.org/ portal/publicaciones-del-clad/revistaclad-reforma-democracia/articulos/015octubre-1999

Kliksberg, B. (1989). Gerencia pública en tiempos de incertidumbre ( $1^{\text {a }} \mathrm{ed}$.). Madrid: Instituto Nacional de Administración Pública.

Koontz, H., Weihrich, H. \& Cannice, M. (2008). Administración. Una perspectiva global y empresarial (13va. ed.). México: McGraw-Hill Interamericana.

Nava, G. (2000). Los estudios de caso en la enseñanza de gerencia pública: uso y preparación. En E. Cabrero \& G. Nava, G. (coord.), Gerencia pública municipal. Conceptos básicos y estudios de caso (1 $1 \mathrm{ra}$. ed., pp. 91-138). México: Centro de Investigación y Docencia Económicas - M. A. Porrúa.

Osborne, D. \& Gaebler, T. (1994). La reinvención del gobierno. La influencia del espíritu empresarial en el sector público. Barcelona: Ediciones Paidós.

Osborne, D. \& Plastrik, P. (1998). La reducción de la burocracia. Cinco estrategias para reinventar el gobierno. Barcelona: Ediciones Paidós.

Paramés, C. (1978). Introducción al management. Madrid: Escuela Nacional de Administración Pública. 
Programa de las Naciones Unidas para el Desarrollo-PNUD. (2004). La Democracia en América Latina. Hacia una democracia de ciudadanas y ciudadanos. Recuperado el 8 de junio de 2011, de http://www. bantaba.ehu.es/obs/ocont/pd/obsdoc/ demamerlat/

Shand, D. (1996). La nueva gestión pública: retos y temas de discusión en una perspectiva internacional. Revista de Administración Pública, 91, 77-88.

Sistema Regional de Información sobre Buenas Prácticas de Gestión Pública en América Latina y El Caribe -SIPAL. (2006). Servicio de Atención al Ciudadano (SAC). Recuperado el 11 de julio de 2011, de http://www.sipalonline.org/practicas/ complete_html/sac.html

Tamayo, M. (1997). El análisis de las políticas públicas. En R. Bañón \& E. Carrilo (comps.). La nueva administración pública (pp. 281-312). Madrid: Alianza Editorial.

Torres, J. (2007). Descripción de las experiencias y resultados obtenidos en el desarrollo de iniciativas de reingeniería de procesos en dos organizaciones estatales mexicanas: Los casos de los puertos de Guaymas y Vallarta. Revista de la Red de Expertos Iberoamericanos en Calidad en la Administración Pública, 1, 18-22. 